Literature:

1) K. Hauffe, "Oxidation of Metals," P. 367 (1965), Prenum Press, New York.

2) J. Gurgey, Kim. Sanayi 11, 81 (1962).
3) L. Backland, P. Backland, Arkiv. Khem. 24, (No. 4), 81 (1965).

4) H. Kobayashi, C. Wagner, J. Chem. Phys. 26, 1609 (1957).

\title{
Potentiodynamic Oxidation of Iron in Neutral Solution.*
}

\author{
Norio SATo and Takenori Notoya \\ Physical Chemistry Laboratory, Faculty of Engineering, \\ Hokkaido University, Sapporo, Japan.
}

Received July 4, 1967

The dynamic characteristics of the anodic oxidation processes of iron, including film growth and oxygen evolution, were investigated by application of a potential sweep at various constant rates to passivated iron in a boric acid-borate neutral solution. The film growth begins as a fast process followed by a slow steady growth, with the initial fast process taking place in two successive steps. It is found that the oxygen evolution always succeeds the oxide film growth.

A three dimensional diagram $(\mathrm{E}-\log i-\Delta Q)$ is presented according to the rate equation $i=k \exp (\beta E-\Delta Q / B)$ to show the transitions from the initial stages of the film growth to the steady thickening of the film and from the film growth to the oxygen evolution. Dependence upon kinetic constants $B$ and $\beta$ of the potential sweep rate is represented as a spectrum in reaction rate for the anodic oxidation of iron, demonstrating that, if the rate of potential sweep is chosen properly, one can measure the initial stages, the steady film growth and the oxygen evolution separately.

\section{Introduction}

Anodic oxidation reactions of passivated iron in neutral solution are complex and consist of oxide film formation and

* Originally written in Japanese in Denki-Kagaku 35 (No. 6), (1967). oxygen evolution. In a previous paper1) Sato and Cohen have studied the oxide film growth on iron in neutral solution using the potentiostatic and galvanostatic oxidation techniques and presented the following rate equations for the initial stages of the film thickening 


$$
i=k^{\prime} \exp \left(\beta^{\prime} E\right),
$$

and for the subsequent steady growth

$$
i=k \exp (\beta E-Q / B)
$$

where $E$ is the electrode potential, $Q$ the film thickness in amount of charge accumulated, and $k, k, \beta^{\prime}, \beta$ and $B$ are kinetic constants. Further investigation of the transitions from the initial stages to the steady growth of the film and from the film formation to the oxygen evolution is necessary in order to elucidate the whole mechanism of anodic oxidation of iron. In this paper some results obtained by means of potentiodynamic oxidation are presented.

Application of a potential that changes linearly with time for measuring currentpotential curves is well known in polarography and initially was employed almost solely in the field of analytical chemistry except for a few instances 2 ),3). In the last few years this method has been develop$\left.\mathrm{ed}^{4}\right)^{15}$ ) as one of the transient methods of electrochemical polarization, and it has often been used recently in the field of metallic corrosion and deposition to measure the so-called potentiodynamic, potentiokinetic or potential-sweep polarization curve, the opposite of the potentiostatic polarization curve. There have been, however, few experiments which enable the meaning of the potential sweep rate, $d E / d t$, to be made clear in complex electrochemical reactions, although a number of theoretical investigations have been reported, on a variety of simplified reaction models 23$), 24), 25$ ). This paper also attempts a demonstration of the effect of the potential sweep rate upon the anodic oxidation of iron.

\section{Experimental}

Boric acid-sodium borate buffer solution $(\mathrm{pH}=8.40)$ was used as the test solution. This was prepared by mixing a sodium borate solution $(23 \mathrm{~g} / l)$ and a boric acid solution $(16 \mathrm{~g} / \mathrm{l})$ in a volume ratio of $1 / 2$, the concentration of which was $0.15 N$. Dissolved oxygen gas was removed by cathodic reduction on a $\mathrm{Pt}$ electrode at c.d. of $0.1 \mathrm{~mA} / \mathrm{cm}^{2}$ for several hours followed by bubbling purified nitrogen gas for two days.

An iron specimen of surface area $10 \mathrm{~cm}^{2}$ was prepared from a sheet of carbonyl iron of $99.9 \%$ purity. The specimen was abraded with emery papers, annealed at $600^{\circ} \mathrm{C}$ in vacuum and electropolished in acetic acid-perchloric acid mixture under the same conditions as mentioned in a previous paper ${ }^{1)}$. Immediately before the experiments the specimen was again electropolished and it was then immersed in the solution in such a way that a part of the specimen handle was over the solution.

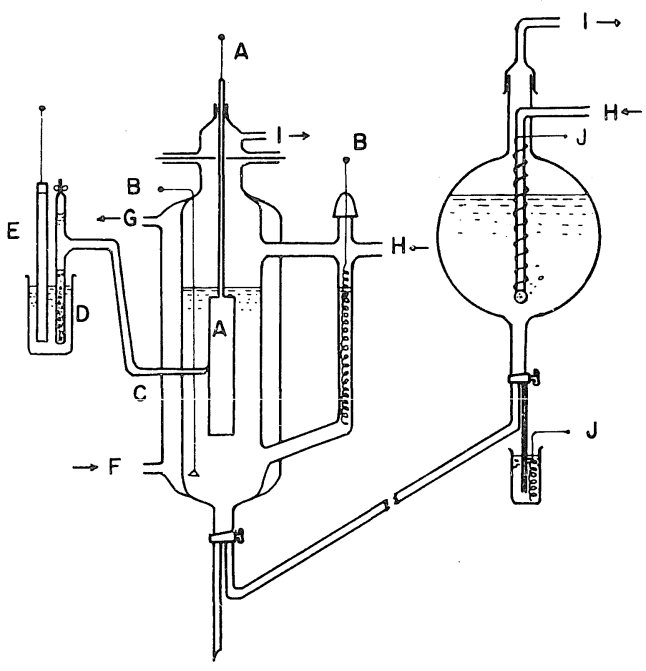

A: Specimen, B: Pt electrode, C: Capillary tip D: Salt bridge, E: Reference electrode (S.C.E.) $F$ and $G$ : Inlet and outlet of purified nitrogen gas,) J; Pt electrode for pre-electrolysis of solution.

Fig. 1 Electrolysis cell. 


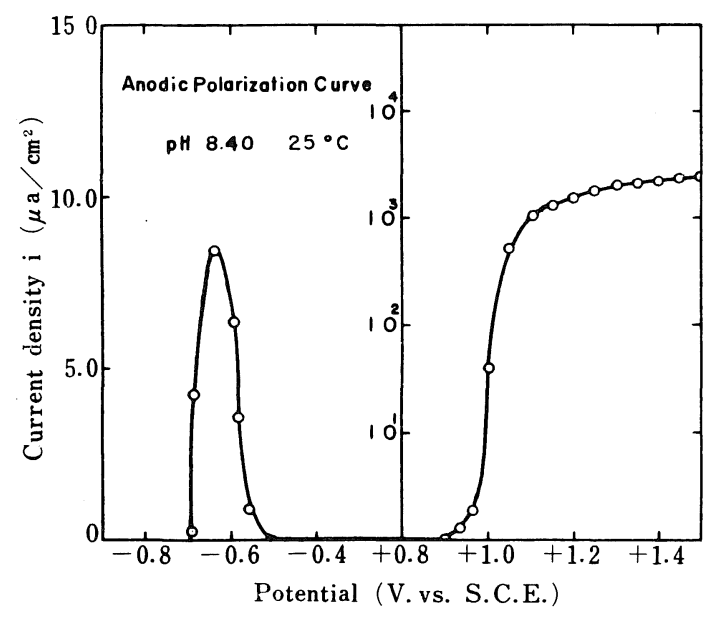

Fig. 2 Nearly stationary polarization curve of iron in a boric acid-borate solution of $\mathrm{pH} 8.40$ at $25^{\circ} \mathrm{C}$.

The electrolytic cell is shown in Fig. 1; it is a cylindrical glass vessel of $100 \mathrm{~m} l$ capacity and is connected to a storage vessel for the solution. A platinum electrode was used as the counter electrode. Electric circuits for polarization measurements consist of a galvanostat and a potentiostat which is connected to a functional voltage generator to apply a potential sweep at various speeds; the potentiostat is of the high-response type having an amplification factor of 1000 , a band width of $50 \mathrm{Kc} / \mathrm{s}$ and a zero-point drift of less than $2 \mathrm{mV}$.

Before measurements the specimen was subjected to a cathodic pretreatment at $10 \mu \mathrm{a} / \mathrm{cm}^{2}$ until the air formed or the passive film was completely reduced, and then the solution was renewed three times to remove the ferrous ion dissolved. All the measurements were perfromed at $25 \pm 0.1^{\circ} \mathrm{C}$ under a nitrogen atmosphere. Electrode potential data reported here all refer to the saturated calomel electrode (S.C.E.).

\section{Results and Discussion}

A nearly stationary polarization curve, which was obtained after $1 \mathrm{hr}$ of anodic polarization at various constant potentials, is shown in Fig. 2. The active state appeares at potentials less noble than $-0.5 \mathrm{~V}$, with a maximum current of 8.4 $\mu \mathrm{a} / \mathrm{cm}^{2}$ at $-0.64 \mathrm{~V}$. In the potential region where passivity occurs the current is less than $0.1 \mu \mathrm{a} / \mathrm{cm}^{2}$. Above a potential of $+0.90 \mathrm{~V}$ the current begins to increase with potential, and evolution of oxygen occurs on the surface of iron. The current

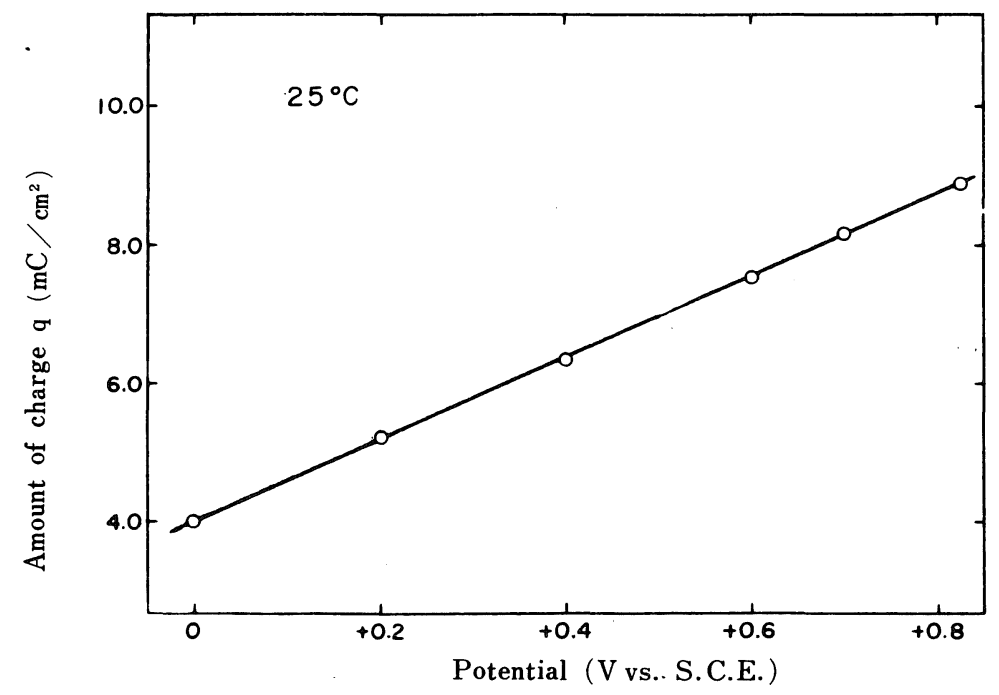

Fig. 3 Amount of total charge Q, accumulated in formation of the passive film during $1 \mathrm{hr}$. oxidation at various potentials. 
is constant beyond a potential of about $+1.10 \mathrm{~V}$. It was found by chemical analysis using the ortho phenanthroline method that here were no ferrous ions in the solution after $1 \mathrm{hr}$ anodic oxidation at potentials in the range from $-0.20 \mathrm{~V}$ to $+1.50 \mathrm{~V}$. The limiting sensitivity of this method was $0.05 \mu \mathrm{g} \mathrm{Fe}$ in the total solution of $50 \mathrm{ml}$.

In Fig. 3 the amount of charge accumulated in the formation of the passive oxide film during $1 \mathrm{hr}$ potentiostatic oxidation is plotted against the potential. The amount of charge has been calculated by integrating graphically the $i-t$ curves obtained. All these curves exhibited initially a large anodic current of the order of $100 \mu \mathrm{A} / \mathrm{cm}^{2}$ followed by a rapid fall in the current towards the order of $1 \mu \mathrm{A} / \mathrm{cm}^{2}$ in about $10 \mathrm{~min}$. The amount of charge thus calculated can be considered to correspond to the film thickness because of the absence of iron ions in the solution after the anodic oxidation. It is

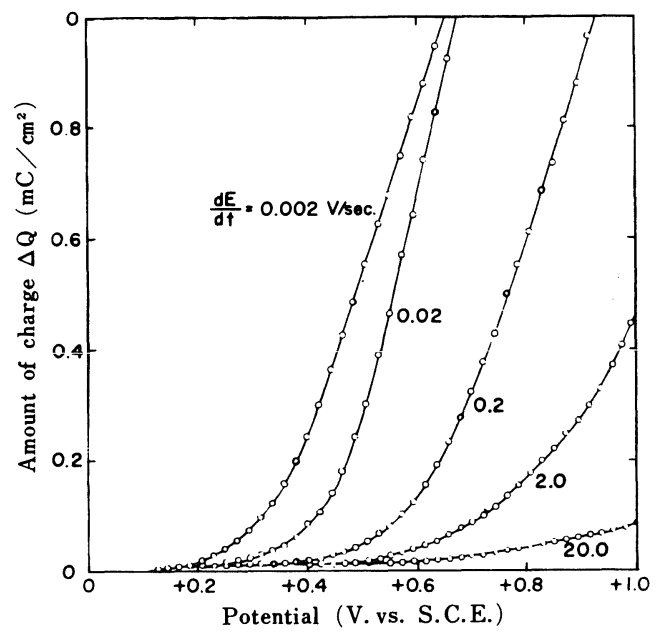

Fig. 4 Relation between potential and amount of charge, $\Delta Q$, passed during potentiodynamic oxidation at various sweep rates.

seen that the amount of charge increases linearly with the applied potential with a slope of $5.96 \mathrm{mC} / \mathrm{V}$.
After anodic oxidation at $+0.0 \mathrm{~V}$ for $1 \mathrm{hr}$ the iron specimen was further oxidized anodically by application of a linear potential sweep from $0.0 \mathrm{~V}$ to $+1.50 \mathrm{~V}$, the rate of which was changed within the range $0.002 \mathrm{~V} / \mathrm{sec}$ to $20 \mathrm{~V} / \mathrm{sec}$, and the amount of charge passed was calculated at every interval during the potentialsweep oxidation in the same way as above; i.e. graphic integration of the measured $i-E$, and hence $i-t$ curves. Some of results obtained but not all avoiding complexity, are shown in Fig. 4, where the amount of charge passed is plotted against potential. It should be noted that at every point on the curves the current is different. At potentials less noble than $+0.9 \mathrm{~V}$ the passage of charge caused further thickening of the passive oxide film formed previously, whereas at potentials more positive than $+0.9 \mathrm{~V}$ it caused both thickening of the film and oxygen evolution.

The dependence of the amount of charge passed, $\Delta Q$, on the anodic current, $\mathrm{i}$, at various constant potentials can be derived from the results of the potentialsweep oxidation at various sweep rates with the aid of $\triangle Q-E$ plots (Fig. 4) in the following way. A vertical line of constant potential intersects a number of the $\Delta Q-E$ curves, giving at each point of intersection a pair of the numerical values of $\Delta Q$ and $i$. The results thus derived at eight successive potentials are shown in Fig. 5. It is noted that for each curve the rate of the potential sweep at a given potential is not constant but decreases with increase of $\Delta Q$, as illustrated by the curve at $+1.0 \mathrm{~V}$. These are the $\log i$ versus $\Delta Q$ curves obtained by means of potential-sweep oxidation and are much the same as those obtained by potentiostatic oxidation in previous papers ${ }^{1), 26)}$ Rate equation (2), which governs the steady growth of the passive film in both po- 
tentiostatic and galvanostatic oxidations, describes also the last part of $\log i$ versus $\triangle Q$ curves obtained potentiodynamically in the passive potential region, where the parameter B is almost independent of the potentials.

On assumption of the validity of rate equation (2) extending to all states of the anodic oxidation in the potential regions of the film growth and oxygen evolution, one can divide the $\log i$ versus $\triangle Q$ curves into four regions with regard to parameter B, as indicated in Fig. 5 .

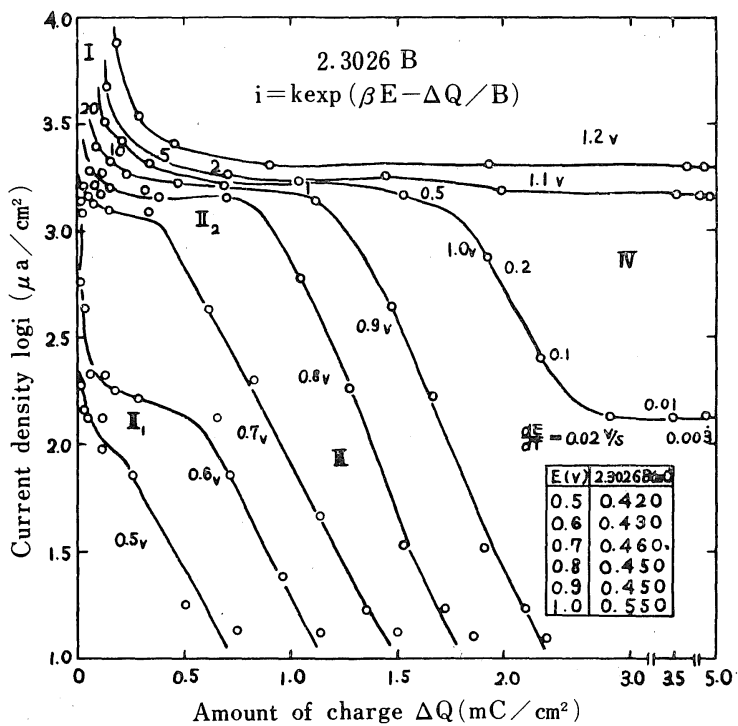

Fig. 5 Log $i$ versus $\Delta Q$ curves obtained potentiodynamically and estimated values of parameter $\beta$ in region III and IV.

Region I which is assigned to very early stages of the anodic oxidation can be detected only at very large rates of the potential sweep. The current in this region may correspond to the charging of the electric double layers. Region II is attributed to the initial stages of thickening of the passive oxide film for which rate equation (1) has been developed and the current is seen almost independent of $\Delta Q$.
The processes occurring in this region have been investigated by one of the authors 1),26). Formation of an iron ion vacancy, oxygen ion excess, or desorption of proton on the outer-layer of the passive oxide film will play a predominant role in this region.

There appears to be a little difference between region $\mathbb{I I}_{1}$ which advances the film growth only and region $\mathbb{I}_{2}$ which is followed by both film growth and oxygen evolution. Region III is assigned to the steady growth of the oxide film which has been discussed in a previous paper ${ }^{1}$, and in region IV oxygen evolution occurs and the current is again almost independent of $\Delta Q$. Successive progress from region I towards region IV is distinctly seen from a curve of $\log i$ versus $\triangle Q$ at $1.00 \mathrm{~V}$ in Fig. 5.

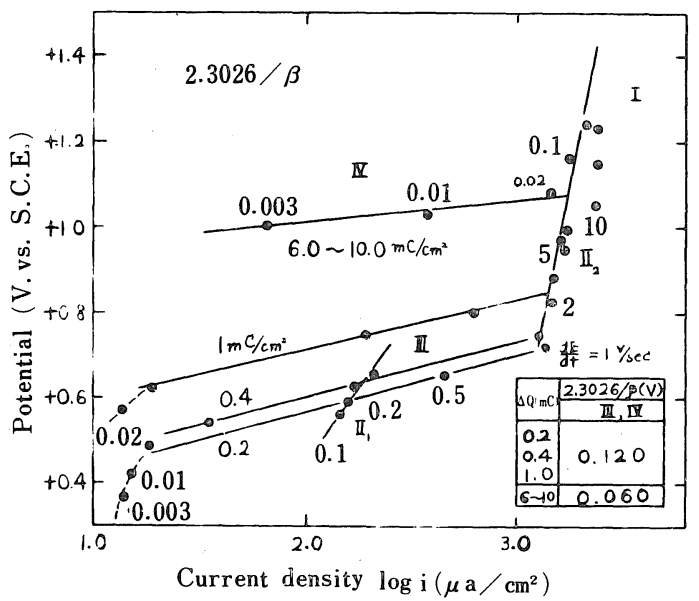

Fig. $6 \quad \mathrm{E}$ versus $\log i$ curves obtained potentiodynamically and estimated values of parameter $\beta$ in region III and IV.

From the results of the potential-sweep oxidation experiments, a current-potential relation at constant amounts of charge passed can be derived in the same way as above; a horizontal line of constant $\Delta Q$ in Fig. 4 intersects a number of the 
$\Delta Q-E$ curves, giving at each point of intersection a pair of $E$ and $i$. The current-potential curves thus obtained at various $\Delta Q$ 's are shown in Fig. 6. These curves provide numerical values of the parameter $\beta$ for each of the four regions mentioned above. There is however a lack of distinction with respect to $\beta$ between region $I$ and region II at large $\Delta Q$ 's. As can be seen in the figure, the current potential curve in region III shifts with a nearly constant Tafel slope of $0.120 \mathrm{~V}$ in the direction of positive potential with increasing $\Delta Q$, giving one Tafel line in each region whose slope approximates $0.06 \mathrm{~V}$ for region $\mathrm{IV}$ and infinity for region II.

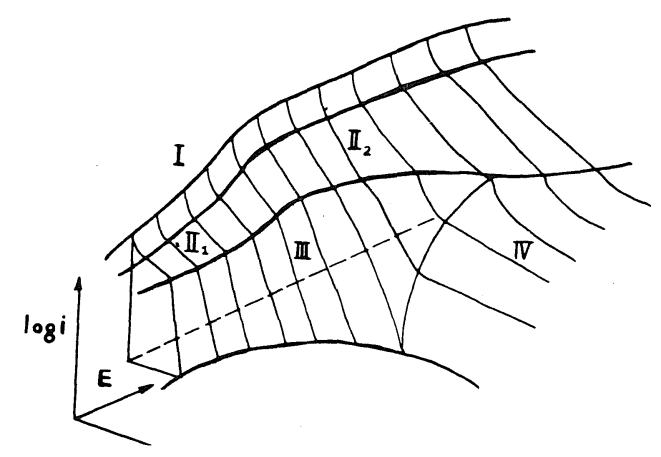

I \& II : Initial stages of the film growth

III: Steady growth of the film

IV: Oxygen evolution

Fig. 7 Schematic representation of three dimensional diagram $(E$-log $i-\Delta Q)$ for anodic oxidation of iron.

Combination of Fig. 5 and Fig. 6 gives a three dimensional diagram with coordinates expressing $E, \mathrm{i}$ and $\Delta Q$, in which all the results in the present experiments can be summarized, as shown schematically in Fig. 7. Thick lines in this figure stand for transitions between the regions and fine lines represent ones of iso-potential. Diagrams of this kind will be very useful to understand the over-all anodic behavior of iron. It is easily seen that the film thickening always precedes the oxygen evolution even in the potential region of oxygen evolution.

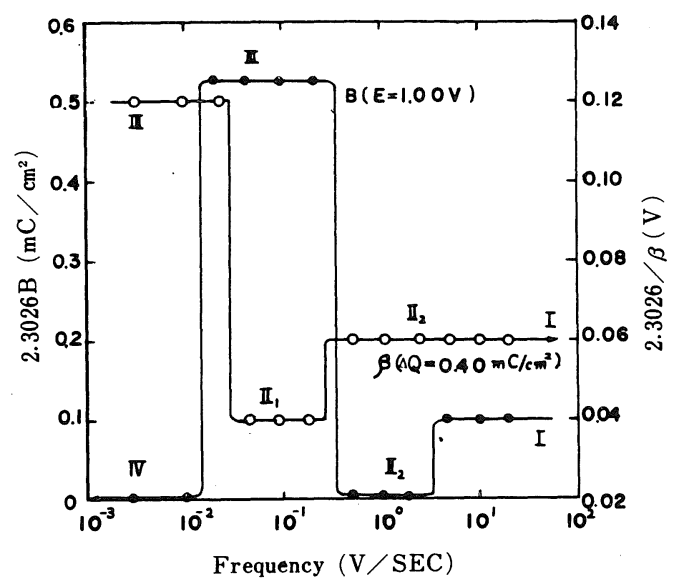

Fig. 8 Dependence of kinetic parameters $\beta$ and $B$ upon the rate of potential sweep.

Dependence of the potential-sweep rate upon the kinetic parameters $B$ and $\beta$ can also be derived with the aid of the two types of plot shown in Fig. 5 and 6 . Parameter $\mathrm{B}$ at $1.00 \mathrm{~V}$ and parameter $\beta$ at $\Delta Q=0.40 \mathrm{mc} / \mathrm{cm}^{2}$ are plotted as a function of the potential sweep rate in Fig. 8. This may be regarded as a spectrum of reaction rate in the form of equation (2) for the anodic oxidation of iron. Although the results requires further study and analysis, such a spectrum could provide information about the rate of elemental steps composing the over-all anodic oxidation reaction of the film growth and oxygen evolution.

\section{Summary}

Anodic oxide film growth and oxygen evolution in a neutral solution were studied by using the potentiodynamic method.

The oxide film growth begins as a fast process comprising two successive 
steps which are followed by a steady film growth process. The oxygen evolution always succeeds the oxide film growth even in the potential region of oxygen liberation.

A three dimentional diagram is presented in a schematic manner to show the relationship between the potential, the current and the amount of charge passed during the anodic oxidation according to the rate equation, $i=k \exp (\beta E-Q / B)$. This diagram serves to distinguish between the regions of the initial processes, the steady growth and the oxygen evolution.

Dependence of the potential sweep rate upon kinetic parameters $B$ and $\beta$ is also represented as a spectrum of the reaction rate. It is suggested that, if the rate of the potential sweep is chosen properly, the initial stages of the film growth, the steady growth process of the oxide film and the oxygen evolution process can be measured separately.

Acknowlegements: The authors wish to thank professor Go Okamoto for discussion.

\section{Litratures:}

. 1) N. Sato, M. Cohen, J. Electrochem, Soc. 111, 512, 519 (1962).

2) P. Delahay, G. Perkins, J. Phys Colloid Chem. 55, 586, 1146 (1951).

3) J. W. Loveland, P. Elving, J. Phys. Chem. 50, 250 (1952).

4) A. L. Juliard, Nature 183, 1040 (1959).
5) F. G. Will, C. A. Knorr, Z. Elektrochem. 64, 258 (1960).

6) W. Boeld, M. Breiter, ibid. 64, 270 (1960).

7) M. W. Breiter, S. Gilman, J. Electrochem. Soc. 109, 622 (1962).

8) R. P. Buck, L. R. Griffith, ibid. 109, 1005 (1962).

9) S. Gilman, J. Phys. Chem. 66, 2657 (1962).

10) A. L. Juliard, H. Shalit, J. Electrochem. Soc. 110, 1002 (1963).

11) M. W.Breiter, Electrochim. Acta 8, 447 (1963)

12) M. W. Breiter, ibid. 8, 925 (1963).

13) R. A. Rightnire, R. L. Rowland, D. L. Boss, D. L. Beals, J. Electrochem. Soc. 111, 232 (1964).

14) F. G. Will, ibid. 112, 451 (1965).

15) D. R. Rodes, E. F. Steigelman, ibid. 112, 16 (1965).

16) C. Edeleanu, J. Iron and Steel Inst. 186, 122 (1958).

17) I. Epelboin, M. Fromet, Ph. Morel, Electrochim. Acta 6, 51 (1962).

18) M. Prazak, Corrosion 19, 75t (1963).

19) Littlewood, Corrosion Science 3,99(1963).

20) N. D. Greene, R. Leonard, Electrochim. Acta 9, 45 (1964).

21) J. Serre, J. Laureys, Corrosion Science 5, 141 (1965).

22) S. Pizzini, L. Agace, ibid. 5, 193 (1965).

23) F. G. Will, J. Electrochem. Soc.112, 1157 (1965).

24) B. E. Conway, J. Electroanal. Chem. 8, 486 (1964).

25) B. E. Conway, E. Gileadi, H. Angerstein Kozlowska, J. Electrochem. Soc. 112, 341 (1965).

26) N. Sato, Electrochim. Acta, 12, 1135 (1967). 